\title{
Ontogenetic symmetry and asymmetry in energetics
}

\section{André M. De Roos}

Johan A.J. (Hans) Metz (metz@iiasa.ac.at)

Lennart Persson

\section{Approved by}

Ulf Dieckmann

Director, Evolution and Ecology Program

February 2015 Institute, its National Member Organizations, or other organizations supporting the work. 


\title{
Ontogenetic symmetry and asymmetry in energetics
}

\author{
André M. De Roos • Johan A. J. Metz • \\ Lennart Persson
}

This article is dedicated to Odo Diekmann, who provided so many of the building blocks for its contents

Submitted: June 28, 2012/ Received: ??? / Accepted: ???

\begin{abstract}
Body size (三 biomass) is the dominant determinant of population dynamical processes such as giving birth or dying in almost all species, with often drastically different behaviour occurring in different parts of the growth trajectory, while the latter is largely determined by food availability at the different life stages. This leads to the question under what conditions unstructured population models, formulated in terms of total population biomass, still do a fair job. To contribute to answering this question we first analyze the conditions under which a size-structured model collapses to a dynamically equivalent unstructured one in terms of total biomass. The only biologically meaningful case where this occurs is when body size does not affect any of the population dynamic processes, this is the case if and only if the mass-specific ingestion rate, the mass-specific biomass production and the mortality rate of the individuals are independent of size, a condition to which we refer as "ontogenetic symmetry". Intriguingly, under ontogenetic symmetry the equilibrium biomass-body size distribution is proportional to 1 /size, a form that has been conjectured for marine size spectra and subsequently has been used as prior assumption in theoretical papers dealing with the latter.

As a next step we consider an archetypical class of models in which reproduction takes over from growth upon reaching an adult body size, in order to determine how quickly discrepancies from ontogenetic symmetry lead to rele-

André M. De Roos

Institute for Biodiversity and Ecosystem Dynamics, University of Amsterdam, The Netherlands. E-mail: A.M.deRoos@uva.nl

Johan (Hans) A. J. Metz

Mathematical Institute \& Institute of Biology \& NCB Naturalis, Leiden, Niels Bohrweg 1, 2333 CA, Leiden, Netherlands. \& EEP, IIASA, Laxenburg, Austria. E-mail: J.A.J.Metz@biology.leidenuniv.nl

Lennart Persson

Department of Ecology and Environmental Science, Umeå University S-901 87 Umeå, Sweden. E-mail: Lennart.Persson@emg.umu.se
\end{abstract}


vant novel population dynamical phenomena. The phenomena considered are biomass overcompensation, when additional imposed mortality leads, rather unexpectedly, to an increase in the equilibrium biomass of either the juveniles or the adults (a phenomenon with potentially big consequences for predators of the species), and the occurrence of two types of size-structure driven oscillations, juvenile-driven cycles with separated extended cohorts, and adult-driven cycles in which periodically a front of relatively steeply decreasing frequencies moves up the size distribution. A small discepancy from symmetry can already lead to biomass overcompensation; size-structure driven cycles only occur for somewhat larger discrepancies.

Keywords physiologically structured population · ontogenetic symmetry · size-structure $\cdot$ biomass overcompensation $\cdot$ population cycles $\cdot$ size spectrum

Mathematics Subject Classification (2000) 92D25

\section{Introduction}

Text books in ecology define population dynamics as the change in density of populations in space and time with density referring only to the number of individuals, without taking into account e.g. their body size (e.g. Begon et al 1996). In classical theory the key processes in the life history of individual organisms driving population dynamics hence include only reproduction and mortality. More recently, population dynamic models have also been formulated that use total population biomass as a descriptor of abundance (Yodzis and Innes 1992); models which subsequently have been used widely to study dynamics of larger foodwebs (McCann et al 1998; Brose et al 2006). In the same textbooks higher dimensional representations of populations generally take the form of matrix models that describe the dynamics of age class or stage densities over time (Caswell 2001), but these models are typically limited to the dynamics of a single population. For interacting populations the 5 majority of ecological models use a single quantity to represent a population, be it the number of individuals in the population or their total biomass. In the remainder of this paper we will refer to such models in terms of a single population quantity as "unstructured".

Actual life histories of individuals comprise more than reproduction and mortality. In particular, ontogenetic development and growth in body size during an individual's life span is an energetic necessity before production of offspring can occur. This increase should minimally amount to a doubling in body size, but exceeds an order of magnitude for the majority of species (De Roos and Persson 2013). Given that growth in body size is necessary before 25 reproduction can take place and that a substantial fraction of all newborn individuals do not survive till the reproductive stage, ontogenetic development, in particular growth in body size, can even be considered the most prominent process in an individual's life history after mortality. 
Physiologically structured population models (PSPMs, Metz and Diek-

30 mann 1986, De Roos 1997) were specifically developed to allow accounting for the full complexity of individual life cycles. After some early work on sizestructured models mainly for cell populations (VonFoerster 1959, Tsuchiya et al 1966, Bell and Anderson 1967; Fredrickson et al 1967; Sinko and Streifer 1967; Bell 1968; Anderson et al 1969, Sinko and Streifer 1969, 1971; Van-

35 Sickle 1977; Murphy 1983) in the wake of McKendrick (1926), the work on general PSPMs got in full swing during the last three decades after their general formulation by Odo Diekmann and collaborators in the mid 80's (summarised in Metz and Diekmann 1986). This has resulted not only in applications of PSPMs to a variety of systems but also in the development of a

40 rigorous mathematical basis for the formulation of these models (Diekmann et al 1998), methods for computation of their steady states (Diekmann et al 2003 ) and for analysis of their stability (Diekmann et al 2010), and special numerical techniques for their time integration (De Roos 1988) and bifurcation analysis (Kirkilionis et al 2001). In contrast to unstructured models, popu-

45 lation dynamics in PSPMs results from a bookkeeping of events in the lives of all individuals making up a population. The actual modeling hence takes place at the individual level as opposed to the population level and consists of formulating mathematical descriptions of processes such as individual growth in body size, fecundity and mortality. Because PSPMs faithfully account for so the biological details and complexity of the individual life cycle they can be considered the appropriate framework to formulate models of ecological interactions, with the level to which these details and complexities are represented in a particular case a matter of scientific judgement.

The contrast between an ecological theory based on unstructured mod-

55 els and the fact that PSPMs allow capturing more complexity and hence are more realistic naturally leads to the question to what extent and in what respect this additional complexity truly matters for our understanding of ecological dynamics. Analysis of PSPMs for size-dependent interactions between consumers and their resource has, for example, revealed that size-dependent

60 competition can induce novel phenomena not found in unstructured models, such as particular types of population cycles (De Roos et al 1990, De Roos and Persson 2003: Diekmann et al 2010). Another phenomenon, with potentially large ecological repercussions, is the occurrence of a positive relationship between the biomass in a particular class of individuals when mortality is in65 creased (De Roos et al 2007, so-called "biomass overcompensation"). What are the general conditions under which such structure-related phenomena occur? More specifically, under what conditions can the dynamics of a size-structured model be described in terms of an ordinary differential equation (ODE) for a single population variable, either the total number of individuals or total 70 population biomass? And, do structure-related phenomena, such as biomass overcompensation and size-structure driven population cycles, occur so widely that it is generally necessary to account for the size structure of populations in order to understand their dynamics? Such questions are central to attempts at unraveling how size-structured population models relate to unstructured ones 
75 and assessing the conditions under which ecological theory based on unstructured models can apply. As a first answer we below derive the conditions for which a generic size-structured model can be simplified to a single differential equation for the total biomass. Next we embed the thus delimited class of models in an archetypical larger family of size-structured models to determine the conditions for the appearance of the just described size-structure dependent population dynamical phenomena.

\section{Ontogenetic symmetry in ingestion, biomass production and loss}

The abstract conditions under which a structured population model can be faithfully represented by a finite system of ODEs are described in (Metz and Diekmann 1991, last paragraph of Section 4). Below these conditions are worked out, with an eye on their interpretation, for the special case where the end result is a single differential equation for the population biomass. The conditions take the form of a set of invariances of the model ingredients.

Let $s$ denote the body size of an individual organism, which we assume to fully characterize its state of development. Growth in body size is determined by the function $g(R, s)$ representing the growth rate in size for an individual of size $s$ living in an environment with food or resource density $R$. We assume that all individuals are born with a fixed size at birth, $s_{b}$. Development of a single individual in isolation hence follows the ODE

$$
\frac{d s}{d t}=g(R, s), \quad s(0)=s_{b}
$$

Individual reproduction is modeled with the function $\beta(R, s)$, representing the fecundity of an individual with body size $s$, experiencing a food density $R$. Similarly, mortality is determined by a function $d(R, s)$, representing the instantaneous mortality rate of such an individual. Finally, to model the feedback of individuals on their resource environment we define the function $I(R, s)$ as the rate at which an individual with body size $s$ ingests the resource at density $R$.

Denote the population size distribution as $c(t, s)$. Then the forgoing specifications of individual life history functions lead to the following set of equations describing the dynamics of the population size distribution and the resource density:

$$
\begin{aligned}
& \frac{\partial c(t, s)}{\partial t}+\frac{\partial g(R, s) c(t, s)}{\partial s}=-d(R, s) c(t, s) \\
& g\left(R, s_{b}\right) c\left(t, s_{b}\right)=\int_{s_{b}}^{\infty} \beta(R, s) c(t, s) d s \\
& \frac{d R}{d t}=G(R)-\int_{s_{b}}^{\infty} I(R, s) c(t, s) d s
\end{aligned}
$$
equations above specifies the autonomous dynamics of the resource in the 
absence of any individuals consuming it. To complete the model specification the set of equations (2) has to be supplemented with initial conditions for the size distribution $c(0, s)$ and resource density $R(0)$, but for the remainder of this paper we shall not pay further attention to these initial conditions or the transient dynamics following from it.

The central question we address in this section is under which conditions the size-structured population model (2) can be reduced to an ODE for a single quantity representing the density of the consumer population in addition to the ODE describing the resource dynamics. Given the importance of body size in individual life history, we ignore the trivial case that all life history functions $g(R, s), \beta(R, s), d(R, s)$ and $I(R, s)$ are independent of body size $s$, when it is straightforward to simplify the model (2) to an unstructured model in terms of the total number of individuals in the population. Instead, we focus on describing the consumer dynamics in terms of the total population biomass $B$, defined as

$$
B(t)=\int_{s_{b}}^{\infty} s c(t, s) d s
$$

Differentiating this expression with respect to time yields after substitution of equation 2a the ODE

$$
\frac{d B}{d t}=-\int_{s_{b}}^{\infty} s \frac{\partial g(R, s) c(t, s)}{\partial s} d s-\int_{s_{b}}^{\infty} s d(R, s) c(t, s) d s
$$

Partial integration of the first integral on the right-hand side of this ODE yields

$$
\begin{aligned}
\frac{d B}{d t}=s_{b} g\left(R, s_{b}\right) c( & \left.t, s_{b}\right)-\lim _{s \rightarrow \infty} s g(R, s) c(t, s) \\
& +\int_{s_{b}}^{\infty} g(R, s) c(t, s) d s-\int_{s_{b}}^{\infty} s d(R, s) c(t, s) d s
\end{aligned}
$$

The second term on the right-hand side of this equation is necessarily equal to 0 in any realistic, ecological model, whereas the first term on the right-hand side can be rewritten using the boundary condition (2b), resulting in

$$
\frac{d B}{d t}=\int_{s_{b}}^{\infty}\left(\frac{s_{b} \beta(R, s)+g(R, s)}{s}-d(R, s)\right) s c(t, s) d s
$$

The above ODE only reduces to a closed equation for $B$ if

$$
\frac{d}{d s}\left(\frac{s_{b} \beta(R, s)+g(R, s)}{s}-d(R, s)\right)=0 \quad \text { for } s \geq s_{b}
$$

This condition is formulated in terms of the balance between the rate at which new biomass is produced per unit biomass through either reproduction or somatic growth, $\left(s_{b} \beta(R, s)+g(R, s)\right) / s$, and the rate at which biomass is destroyed through mortality, $d(R, s)$. Since there is little reason to suppose a close coupling between the biological mechanisms underlying an individual's 
mass-specific production of new biomass and the mortality to which it is exposed, we shall in the following focus on conditions on these terms separately that together guarantee (6). In addition, to be able also to write the ODE (2c) for the resource dynamics in terms of $R$ and $B$ the ingestion rate per unit biomass, $I(R, s) / s$, should be independent of individual body size as well.

Reduction of the size-structured consumer-resource model (2) to a coupled set of ODEs for resource and consumer biomass is hence possible if the following three conditions hold:

Ingestion invariance: the mass-specific rate of resource ingestion is independent of body size

$$
\frac{\partial}{\partial s}\left(\frac{I(R, s)}{s}\right)=0 \quad \text { for } s \geq s_{b}
$$

Biomass production invariance: the mass-specific rate at which new biomass is produced is independent of body size

$$
\frac{\partial}{\partial s}\left(\frac{s_{b} \beta(R, s)+g(R, s)}{s}\right)=0 \quad \text { for } s \geq s_{b}
$$

Mortality invariance: the mortality rate is independent of body size

$$
\frac{\partial}{\partial s} d(R, s)=0 \quad \text { for } s \geq s_{b}
$$

The three invariance conditions determine a symmetry between individuals of different body sizes in mass-specific ingestion, mass-specific biomass production and mortality, respectively, to which we refer together as ontogenetic symmetry. If there is such ontogenetic symmetry the size-structured dynamics can be simplified to

$$
\begin{aligned}
& \frac{d B}{d t}=h(R) B-d(R) B \\
& \frac{d R}{d t}=G(R)-f(R) B
\end{aligned}
$$

with $h(R)=\left(s_{b} \beta(R, s)+g(R, s)\right) / s$ and $f(R)=I(R, s) / s$, which only dependent on $R$ by assumption. Given the assumption of ontogenetic symmetry we furthermore drop the dependence on body size from the mortality rate $d(R)$. This system of ODEs resembles a classical, unstructured model and becomes with specific choices for $h(R), d(R), G(R)$ and $f(R)$ in fact identical to the model proposed by Yodzis \& Innes $(1992)$, which has recently been used widely to study dynamics of foodwebs (McCann et al 1998; Brose et al 2006). Ontogenetic symmetry in mass-specific ingestion, mass-specific biomass production and mortality is therefore a useful starting point to compare unstructured models with size-structured models, as under these conditions they are dynamically equivalent. It provides the natural reference point for answering the question how quickly deviations from ontogenetic symmetry lead to novel dynamic phenomena that are induced by the population size structure and hence how quickly predictions from size-structured and unstructured population models start to deviate. 


\section{Equilibrium characteristics under ontogenetic symmetry}

In this section we consider the characteristics of the population equilibrium in the case of ontogenetic symmetry and in particular the consequences of ontogenetic symmetry for the population size structure. We make the ecologically plausible assumption that consumer life histories are composed of a juvenile stage with $s_{b} \leq s<s_{m}$, for which $\beta(R, s)=0$ and an adult stage with $s \geq s_{m}$ for which $\beta(R, s)>0$. The size threshold $s_{m}$ represents the body size at maturation.

From 10 a it follows that the equilibrium resource density $\tilde{R}$ satisfies the identity

$$
h(\tilde{R})=d(\tilde{R})
$$

This condition makes clear that at equilibrium the mass-specific production rate of new biomass equals the biomass loss rate. (8) and (9) then lead to the ecologically important conclusion that under conditions of ontogenetic symmetry in equilibrium the mass-specific turnover of biomass is 0 over any size range of consumers. Among others, this implies that both the juvenile and the adult stage are zero net-producing stages in any consumer equilibrium, that is, losses through mortality in each stage exactly equal the production of new biomass through either growth or reproduction.

An expression for the population size distribution at equilibrium, which we denote with $\tilde{c}(s)$, can be derived from (2a):

$$
\tilde{c}(s)=\frac{g\left(\tilde{R}, s_{b}\right) \tilde{c}\left(s_{b}\right)}{g(\tilde{R}, s)} \exp \left(-\int_{s_{b}}^{s} \frac{d(\tilde{R}, \xi)}{g(\tilde{R}, \xi)} d \xi\right)
$$

(see e.g. Metz and Diekmann 1986 De Roos 1997). Let $\tilde{b}$ represent the birth rate in terms of number of individuals in equilibrium:

$$
\tilde{b}=g\left(\tilde{R}, s_{b}\right) \tilde{c}\left(s_{b}\right)
$$

Under conditions of ontogenetic symmetry the size distribution for juveniles simplifies to

$$
\begin{aligned}
\tilde{c}(s) & =\frac{\tilde{b}}{g(\tilde{R}, s)} \exp \left(-\int_{s_{b}}^{s} \frac{d(\tilde{R}, s)}{g(\tilde{R}, s)} d s\right) \\
& =\frac{\tilde{b}}{d(\tilde{R}) s} \exp \left(-\int_{s_{b}}^{s} \frac{1}{\xi} d \xi\right) \quad \Rightarrow \\
\tilde{c}(s) & =\frac{s_{b} \tilde{b}}{d(\tilde{R})} \frac{1}{s^{2}}
\end{aligned}
$$

The product $s_{b} \tilde{b}$ in the above expression can be interpreted as the production rate of new biomass through reproduction by the adult individuals. Total juvenile biomass in equilibrium, $\tilde{J}$, then equals

$$
\tilde{J}=\int_{s_{b}}^{s_{m}} s \tilde{c}(s) d s=\frac{s_{b} \tilde{b}}{d(\tilde{R})} \ln \left(\frac{s_{m}}{s_{b}}\right)
$$


More generally, the biomass in any juvenile size range between $s_{1}$ and $s_{2}$ equals 215

$$
\int_{s_{1}}^{s_{2}} s \tilde{c}(s) d s=\frac{s_{b} \tilde{b}}{d(\tilde{R})} \ln \left(\frac{s_{2}}{s_{1}}\right)
$$

To derive an expression for the adult biomass at equilibrium, we make the rather general and biologically plausible assumption that individuals after maturation invest a size-dependent fraction $\kappa(s)$ of their net production of new biomass into somatic growth, while investing the remaining fraction $1-\kappa(s)$ of this production into reproduction. The reproduction rate in terms of biomass of an adult individual is then related to its somatic growth rate by:

$$
\frac{s_{b} \beta(R, s)}{g(R, s)}=\frac{1-\kappa(s)}{\kappa(s)}
$$

Under conditions of ontogenetic symmetry we can then derive from (8), (11) 225 and (17) that in equilibrium

$$
\frac{d(\tilde{R})}{g(\tilde{R}, s)}=\frac{1}{\kappa(s) s}
$$

and for the size distribution of adult individuals:

$$
\begin{aligned}
& \tilde{c}(s)=\frac{g\left(\tilde{R}, s_{m}\right) \tilde{c}\left(s_{m}\right)}{g(\tilde{R}, s)} \exp \left(-\int_{s_{m}}^{s} \frac{d(\tilde{R}, s)}{g(\tilde{R}, s)} d s\right) \Rightarrow \\
& \tilde{c}(s)=\frac{s_{b} \tilde{b}}{d(\tilde{R})} \frac{s_{m}^{-1}}{\kappa(s) s} \exp \left(-\int_{s_{m}}^{s} \frac{1}{\kappa(\xi) \xi} d \xi\right)
\end{aligned}
$$

$$
\text { to the }
$$

in which $g\left(\tilde{R}, s_{m}\right) \tilde{c}\left(s_{m}\right)$ represents the rate at which individuals are recruited to the adult stage (cf. 12 ). This rate is related to the population birth rate $s_{b} \tilde{b}=s_{m} g\left(\tilde{R}, s_{m}\right) \tilde{c}\left(s_{m}\right)$

because the condition of ontogenetic symmetry ensures that the juvenile stage is a zero net-production stage of new biomass. The rate at which biomass is recruited to the adult stage therefore equals the rate at which adult individuals produce new biomass through reproduction. The total adult biomass is now given by:

$$
\tilde{A}=\frac{s_{b} \tilde{b}}{d(\tilde{R})} \int_{s_{m}}^{\infty} \frac{s_{m}^{-1}}{\kappa(s)} \exp \left(-\int_{s_{m}}^{s} \frac{1}{\kappa(\xi) \xi} d \xi\right) d s
$$

Partial integration of the integral on the right-hand side leads to

$$
\begin{aligned}
\tilde{A} & =\frac{s_{b} \tilde{b}}{d(\tilde{R})}\left(-\left.\frac{s}{s_{m}} \exp \left(-\int_{s_{m}}^{s} \frac{1}{\kappa(\xi) \xi} d \xi\right)\right|_{s_{m}} ^{\infty}+s_{m}^{-1} \int_{s_{m}}^{\infty} \exp \left(-\int_{s_{m}}^{s} \frac{1}{\kappa(\xi) \xi} d \xi\right) d s\right) \\
& =\frac{s_{b} \tilde{b}}{d(\tilde{R})}\left(1+s_{m}^{-1} \int_{s_{m}}^{\infty} \exp \left(-\int_{s_{m}}^{s} \frac{1}{\kappa(\xi) \xi} d \xi\right) d s\right)
\end{aligned}
$$


where we have used that

$$
\lim _{s \rightarrow \infty} s \exp \left(-\int_{s_{m}}^{s} \frac{1}{\kappa(\xi) \xi} d \xi\right)
$$

is necessarily 0 in any realistic, ecological model. (A mathematical expression of this requirement of biological realism could be that $\lim _{s \rightarrow \infty} \kappa(s)<1$.)

The relative size distribution in equilibrium, $\tilde{c}_{r}(s)$, which we define as the ratio between the equilibrium size distribution $\tilde{c}(s)$ and the total biomass at equilibrium $\tilde{B}=\tilde{J}+\tilde{A}$, then follows from $14,, 15,, 19$ and 22 , resulting in:

$$
\tilde{c}_{r}(s)= \begin{cases}\Phi^{-1} s^{-2} & \text { for } s_{b} \leq s \leq s_{m} \\ \Phi^{-1} \frac{s_{m}^{-1}}{\kappa(s) s} \exp \left(-\int_{s_{m}}^{s} \frac{1}{\kappa(\xi) \xi} d \xi\right) & \text { for } s>s_{m}\end{cases}
$$
in mortality applies. Using (12) we can express the total juvenile biomass in equilibrium $\tilde{J}$, as

$$
\begin{aligned}
\tilde{J} & =\int_{s_{b}}^{s_{m}} s \tilde{c}(s) d s \\
& =g\left(\tilde{R}, s_{b}\right) \tilde{c}\left(s_{b}\right) \int_{s_{b}}^{s_{m}} \frac{s}{g(\tilde{R}, s)} \exp \left(-\int_{s_{b}}^{s} \frac{d(\tilde{R})}{g(\tilde{R}, \xi)} d \xi\right) d s
\end{aligned}
$$

Under conditions of ontogenetic symmetry the consumer biomass at equilibrium, $s \tilde{c}(s)$, thus follows over the juvenile size range a power law as a function of body size, more in particular, is proportional to $s^{-1}$ (cf. equation (23)). This implies that the biomass within logarithmically spaced size groups is constant over the juvenile size range, which interestingly corresponds to a conjecture by Sheldon et al (1972) about the scaling of the size spectrum in the marine environment, and which in previous studies of community size spectra has been assumed prior to analysis (Andersen and Beyer 2006). Here we identify this assumption with the condition of ontogenetic symmetry. From equation (23) we furthermore conclude that the relative size distribution in equilibrium only depends on the size at birth $s_{b}$, the size at maturation $s_{m}$ and the fraction of biomass production that adults invest in somatic growth $\kappa(s)$. Most importantly, this implies that changes in the environment that consumers experience, such as changes in mortality or changes in the productivity of resource biomass $G(R)$, will not affect the relative consumer population composition. In particular, the ratio of juvenile and adult biomass in equilibrium is under conditions of ontogenetic symmetry independent of the mortality rate $d(\tilde{R})$.

Now consider the more relaxed condition when only ontogenetic symmetry 


$$
\tilde{J}=\frac{g\left(\tilde{R}, s_{b}\right) \tilde{c}\left(s_{b}\right)}{d(\tilde{R})}\left(s_{b}-s_{m} \exp \left(-\int_{s_{b}}^{s_{m}} \frac{d(\tilde{R})}{g(\tilde{R}, s)} d s\right)+\int_{s_{b}}^{s_{m}} \exp \left(-\int_{s_{b}}^{s} \frac{d(\tilde{R})}{g(\tilde{R}, \xi)} d \xi\right) d s\right)
$$

Similarly, the adult biomass in equilibrium can be expressed as:

$$
\begin{aligned}
\tilde{A} & =\int_{s_{m}}^{\infty} s \tilde{c}(s) d s \\
& =g\left(\tilde{R}, s_{m}\right) \tilde{c}\left(s_{m}\right) \int_{s_{m}}^{\infty} \frac{s}{g(\tilde{R}, s)} \exp \left(-\int_{s_{m}}^{s} \frac{d(\tilde{R})}{g(\tilde{R}, \xi)} d \xi\right) d s
\end{aligned}
$$

280

with $g\left(\tilde{R}, s_{m}\right) \tilde{c}\left(s_{m}\right)$ representing the rate at which individuals recruit to the adult stage. Partial integration in this case results in

$$
\tilde{A}=\frac{g\left(\tilde{R}, s_{m}\right) \tilde{c}\left(s_{m}\right)}{d(\tilde{R})}\left(s_{m}+\int_{s_{m}}^{\infty} \exp \left(-\int_{s_{m}}^{s} \frac{d(\tilde{R})}{g(\tilde{R}, \xi)} d \xi\right) d s\right)
$$

As before this equation has been simplified using the fact that

$$
\lim _{s \rightarrow \infty} s \exp \left(-\int_{s_{b}}^{s} \frac{d(\tilde{R})}{g(\tilde{R}, s)} d s\right)=0
$$

Using 12 once more to relate $g\left(\tilde{R}, s_{m}\right) \tilde{c}\left(s_{m}\right)$ to the population birth rate in equilibrium, $g\left(\tilde{R}, s_{b}\right) \tilde{c}\left(s_{b}\right)$, then yields the following expression for the ratio between juvenile and adult biomass in equilibrium:

$$
\frac{\tilde{J}}{\tilde{A}}=\frac{s_{b} \exp \left(\int_{s_{b}}^{s_{m}} \frac{d(\tilde{R})}{g(\tilde{R}, s)} d s\right)-s_{m}+\int_{s_{b}}^{s_{m}} \exp \left(\int_{s}^{s_{m}} \frac{d(\tilde{R})}{g(\tilde{R}, \xi)} d \xi\right) d s}{s_{m}+\int_{s_{m}}^{\infty} \exp \left(-\int_{s_{m}}^{s} \frac{d(\tilde{R})}{g(\tilde{R}, \xi)} d \xi\right) d s}
$$

In the case of ontogenetic symmetry both in net production of new biomass and mortality and when adults invest a fraction $\kappa(s)$ of their new biomass production into growth 27 can be shown to simplify to:

$$
\frac{\tilde{J}}{\tilde{A}}=\frac{\ln \left(\frac{s_{m}}{s_{b}}\right)}{1+s_{m}^{-1} \int_{s_{m}}^{\infty} \exp \left(-\int_{s_{m}}^{s} \frac{1}{\kappa(\xi) \xi} d \xi\right) d s}
$$

as can also be derived from 15 and 22 .

If adults do not grow at all the adult biomass in equilibrium equals

$$
\tilde{A}=\frac{s_{m} g\left(\tilde{R}, s_{m}\right) \tilde{c}\left(s_{m}\right)}{d(\tilde{R})}
$$


in which the numerator represents the rate at which biomass is recruited to the adult stage through maturation and $1 / d(\tilde{R})$ equals the average survival time of this biomass. Comparing (26) and (28) shows that the integral in the denominator of (27) is related to the increase in adult biomass due to somatic growth of adults. When adults do not grow in body size (27) simplifies to:

$$
\frac{\tilde{J}}{\tilde{A}}=\frac{s_{b}}{s_{m}} \exp \left(\int_{s_{b}}^{s_{m}} \frac{d(\tilde{R})}{g(\tilde{R}, s)} d s\right)-1+s_{m}^{-1} \int_{s_{b}}^{s_{m}} \exp \left(\int_{s}^{s_{m}} \frac{d(\tilde{R})}{g(\tilde{R} \xi)} d \xi\right) d s
$$

Both (27) and (29) are increasing functions of the mortality-growth rate ratio, $d(\tilde{R}) / g(\tilde{R}, s)$, in the juvenile size range $s_{b} \leq s \leq s_{m}$. This shows that deviations from ontogenetic symmetry that will increase $d(\tilde{R}) / g(\tilde{R}, s)$ for juveniles will increase the juvenile biomass relative to the adult biomass in the population, when compared to the ratio at ontogenetic symmetry. Deviations from ontogenetic symmetry that make the juvenile stage a net loss stage of biomass in equilibrium will therefore lead to a higher juvenile biomass in the population than expected in the case of ontogenetic symmetry. Vice versa, if the juvenile stage becomes a net gain stage of biomass in equilibrium the juvenile biomass will be lower than expected under ontogenetic symmetry.

\section{Novel phenomena through ontogenetic asymmetry}

If ontogenetic symmetry implies that the equilibrium size structure of the population is independent of external factors like an imposed size-independent additional mortality, the question arises how quickly breaking of this symmetry leads to changes of the size structure in response to such a mortality. In physics symmetry breaking gives rise to two differentiated states that are stable in the face of small perturbations starting from a single, disorderly state that is unstable in the face of such perturbations. Similarly, deviations from ontogenetic symmetry lead to either positive relationships between juvenile biomass and mortality or adult biomass and mortality (De Roos et al 2007), with the corresponding adult and juvenile biomasses showing the decrease expected from the symmetric case. We shall refer to such an unexpected increase of biomass with mortality as biomass overcompensation. In this section we investigate how quickly deviations from ontogenetic symmetry give rise to such overcompensation. To this end we focus on a specific size-structured model, in which the ingestion rate, growth rate and fecundity are based on simple energy budget considerations.

Assume that individuals do not grow after maturing at size $s=s_{m}$ and thereafter invest their entire biomass production in reproduction. For simplicity we assume ingestion of resources to be proportional to body size and to follow a Holling type II functional response with an upper mass-specific ingestion rate $M$ and half-saturation density $H$. For juveniles this functional response is multiplied with a factor $(2-q)$ and for adults with $q$. The parameters $q$ hence measures the extent of ontogenetic asymetry in ingestion with 
$q=1$ representing symmetry. In particular, ingestion of resources per unit biomass is modeled for juveniles as

$$
I_{J}(R)=(2-q) \omega(R)
$$

and for adults as

$$
I_{A}(R)=q \omega(R)
$$

in which $\omega(R)=M R /(H+R)$. Ingested resources are assumed to be assimilated into new biomass with a conversion efficiency $\sigma$. Assimilated mass is first used to cover maintenance costs, which are assumed to be proportional to body size as well and amount to $T s$. The net production rate of new biomass per unit juvenile and adult biomass hence equals

$$
\nu_{J}(R)=\sigma I_{J}(R)-T
$$

and

$$
\nu_{A}(R)=\sigma I_{A}(R)-T,
$$

respectively. Notice that by using an energy budget model to couple growth and fecundity to ingestion, we also couple ontogenetic symmetry/asymmetry in production of new biomass directly to ontogenetic symmetry/asymmetry in ingestion. We hence from now on only distinguish between ontogenetic symmetry/asymmetry in either mass-specific biomass production or mortality.

As long as $\nu_{J}(R)$ and $\nu_{A}(R)$ are positive, the somatic growth rate of juveniles equals $\nu_{J}(R) s$, while the adult fecundity equals $\nu_{A}(R) s_{m} / s_{b}$, given that all adults have the same size $s_{m}$ at which they matured and $s_{b}$ is the mass of a single offspring individual (we assume that all overheads to produce offspring have been subsumed in the conversion parameter $\sigma$ ). However, if $\nu_{J}(R)<0$ we assume somatic growth of juveniles to stop, hence

$$
g(R, s)=\max \left(\nu_{J}(R) s, 0\right)
$$

Similarly, we assume reproduction to stop when $\nu_{A}(R)$ is negative:

$$
\beta\left(R, s_{m}\right)=\max \left(\nu_{A}(R) \frac{s_{m}}{s_{b}}, 0\right)
$$

Finally, we assume background mortality to be constant within the juvenile and adult stages, but potentially to differ between these two stages. When $\nu_{J}(R)<0$, juveniles are moreover assumed to suffer from an additional starvation mortality equal to $-\nu_{J}(R)$. Similarly, when $\nu_{A}(R)<0$, adults suffer starvation mortality equal to $-\nu_{A}(R)$. (This is admittedly a fudge, but among the possible choices for this hard to determine relationship it has the singular virtue that it extends the overall mass balances of the model also into the 
starvation regime; see De Roos et al 2008). Juvenile and adult mortality hence follow

$$
d_{J}(R)=(2-p) \mu-\min \left(\nu_{J}(R), 0\right)
$$

and

$$
d_{A}(R)=p \mu-\min \left(\nu_{A}(R), 0\right),
$$

respectively. The parameter $p$ measures the extent of asymmetry in background (not starvation) mortality, with $p=1$ representing ontogenetic symmetry (in direct similarity to our symmetry breaking assumption for the massspecific biomass production rate). Under conditions of ontogenetic symmetry in mortality both juveniles and adults hence have a background mortality rate equal to $\mu$ when they are not starving. Finally, we assume the resource in absence of consumers to follow semi-chemostat dynamics:

$$
G(R)=\rho\left(R_{\max }-R\right)
$$

Based on the above assumptions we can formulate the population model as

$$
\begin{aligned}
& \frac{\partial c(t, s)}{\partial t}+\frac{\partial g(R, s) c(t, s)}{\partial s}=-d_{J}(R) c(t, s) \quad \text { for } s_{b} \leq s<s_{m} \\
& g\left(R, s_{b}\right) c\left(t, s_{b}\right)=\beta\left(R, s_{m}\right) C_{A} \\
& \frac{d C_{A}}{d t}=g\left(R, s_{m}\right) c\left(t, s_{m}\right)-d_{A}(R) C_{A} \\
& \frac{d R}{d t}=G(R)-\left(I_{J}(R) \int_{s_{b}}^{s_{m}} s c(t, s) d s+I_{A}(R) s_{m} C_{A}\right)
\end{aligned}
$$

Because adults do not grow in body size, the PDE (39a) only describes the changes in the juvenile size distribution, whereas the dynamics of the total density of adults, $C_{A}$, is governed by the ODE (39c), reflecting the balance between the rate at which adults are recruited, that is, juveniles mature, $\left(R, s_{m}\right) c\left(t, s_{m}\right)$, and adults die.

We studied the dynamics of the full model (39), including starvation mortality, using numerical methods specifically adapted for dealing with physiologically structured population models along the lines of De Roos (1988). As default values of the parameters we assumed $s_{b}=0.1, s_{m}=1.0, M=1.0$, $H=3.0, T=0.1, \mu=0.015, \sigma=0.5, \rho=0.1$ and $R_{\max }=100.0$ (De Roos and Persson 2013). (Note that by scaling it can be seen that any qualitative model predictions depend only on the dimensionless parameters $z=s_{b} / s_{m}$, $M / T, \mu / T, \rho / T$ and $R_{\max } / H$ and $\sigma$, cf. De Roos et al 2008). In addition, the model contains two dimensionless symmetry parameters, $p$ and $q$, representing the asymmetry in mortality and mass-specific biomass production, respectively. The default values for both symmetry parameters are 1 , but our 
main interest is the effects of variations in these symmetry parameters on any model predictions.

For analyzing changes in the population size structure at equilibrium with mortality, the model can be simplified since the equilibrium resource density is necessarily high enough to prevent starvation. By continuity the same holds good for a small neighborhood of the equilibrium, so that starvation can also be ignored when analyzing its stability. In the absence of starvation $g(R, s)=$ $\nu_{J}(R) s, \beta(R, s)=\nu_{A}(R) s_{m} / s_{b}, d_{J}=(2-p) \mu$ and $d_{A}=p \mu$. (Notice that we here drop the dependence of the mortality rates on resource density as they are constant as long as starvation does not occur.) These simplifications allow the model to be reformulated as a system of delay-differential equations in terms of the juvenile biomass:

$$
J(t)=\int_{s_{b}}^{s_{m}} s c(t, s) d s
$$

and the adult biomass $A(t)=s_{m} C_{A}(t)$, respectively. Following the procedures described in Nisbet and Gurney (1983), this results in, with $z=s_{b} / s_{m}$ the net size increase from birth to maturation,

$$
\begin{aligned}
\frac{d J}{d t} & =\nu_{A}(R(t)) A(t)+\nu_{J}(R(t)) J(t)-d_{J} J(t)-\Psi(t) \\
\frac{d A}{d t} & =\Psi(t)-d_{A} A \\
\frac{d R}{d t} & =G(R)-I_{J}(R) J(t)-I_{A}(R) A(t) \\
\Psi(t) & =\frac{\nu_{A}(R(t-\tau(t))) A(t-\tau(t)) \nu_{J}(R(t))}{z \nu_{J}(R(t-\tau(t)))} e^{-d_{J} \tau(t)} \\
-\ln (z) & =\int_{t-\tau(t)}^{t} \nu_{J}(R(\xi)) d \xi
\end{aligned}
$$

The ratio $\nu_{J}(R(t)) / \nu_{J}(R(t-\tau(t)))$ in the expression for the maturation $\Psi(t)$ (41d) accounts for the effect of the variation in specific growth at the times $t$ and $t-\tau(t)$, where $\tau(t)$ is the time delay between birth and maturation, with $z$ the, fixed, biomass increase during that time. Equation $41 \mathrm{e}$ determines the time delay $\tau(t)$, given that juveniles after birth grow in body size at a specific rate $\nu_{J}(R)$.

To find the equilibrium we first derive from Equation $41 \mathrm{e}$ an expression for the time delay between birth and maturation for constant resource density:

$$
\tilde{\tau}=-\ln (z) / \nu_{J}(\tilde{R})
$$

Furthermore, we combine Equations $41 \mathrm{~b}$ and $41 \mathrm{~d}$ to

$$
d_{A}=\frac{\nu_{A}(\tilde{R})}{z} e^{-d_{J} \tilde{\tau}}
$$




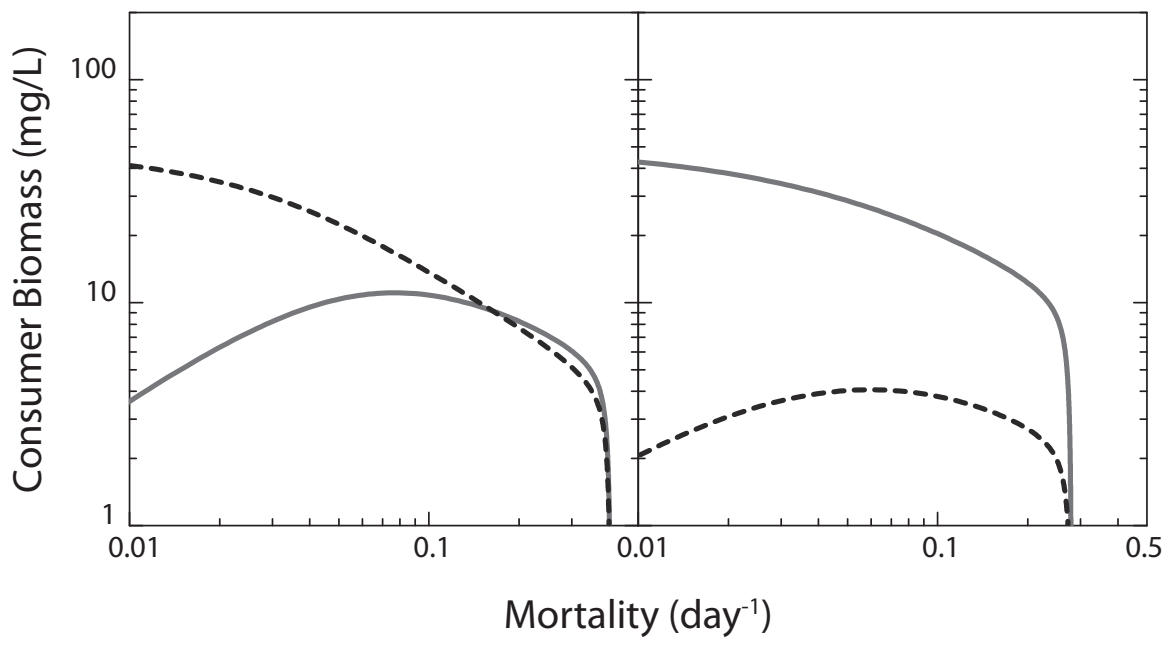

Fig. 1 Biomass overcompensation in juvenile (left, $q=0.65, p=1.0$ ) and adult biomass (right, $q=1.35, p=1.0)$ in response to mortality $\left(d_{J}+m=d_{A}+m=\mu+m\right)$. Solid lines: juvenile biomass, dashed lines: adult biomass.

Finally, we combine $(42)$ and $(43)$ into an equation for determining the equilibrium resource density $\tilde{R}$ :

$$
\frac{\nu_{A}(\tilde{R}) z^{d_{J} / \nu_{J}(\tilde{R})-1}}{d_{A}}=1
$$

The left-hand side of (44) represents the expected number of offspring that a consumer produces over a life time, which obviously at equilibrium has to be equal to 1.

From Equation 41a and 41b we can furthermore derive that juvenile and adult biomass at equilibrium are related to each other as

$$
\tilde{J}=-\frac{\nu_{A}(\tilde{R})-d_{A}}{\nu_{J}(\tilde{R})-d_{J}} \tilde{A},
$$

which together with Equation (41c) yields the following expressions for juvenile and adult biomass at equilibrium:

$$
\begin{aligned}
& \tilde{J}=\frac{G(\tilde{R})\left(\nu_{A}(\tilde{R})-d_{A}\right)}{I_{J}(\tilde{R})\left(\nu_{A}(\tilde{R})-d_{A}\right)-I_{A}(\tilde{R})\left(\nu_{J}(\tilde{R})-d_{J}\right)} \\
& \tilde{A}=\frac{G(\tilde{R})\left(\nu_{J}(\tilde{R})-d_{J}\right)}{I_{A}(\tilde{R})\left(\nu_{J}(\tilde{R})-d_{J}\right)-I_{J}(\tilde{R})\left(\nu_{A}(\tilde{R})-d_{A}\right)}
\end{aligned}
$$

Equations (46) and (47) give the equilibrium juvenile and adult consumer biomasses as functions of the equilibrium resource biomass, with the latter determined by the transcendental Equation (44). Studying the response of these 
equilibrium biomass densities to additional mortality can be achieved by replacing in equations (44), (46) and (47) the juvenile and adult mortality, $d_{J}$ and $d_{A}$, with $d_{J}+m$ and $d_{A}+m$, respectively, and varying $m$. For the case $p=1$, that is, $d_{J}=d_{A}=\mu$, Figure 1 shows examples of the dependence of the juvenile and adult biomass at equilibrium on the total consumer mortality $d_{J}+m=d_{A}+m=\mu+m$. When $q<1$, that is, when juvenile consumers have a higher mass-specific biomass production rate than adults, adults dominate the population biomass at low mortality. This corresponds to our conclusion at the end of the previous section that in the case that the juvenile stage is a net gain stage of biomass in equilibrium juvenile biomass makes up a smaller proportion of total biomass than expected when ontogenetic symmetry applies. Increasing mortality then leads to a change in population size structure such that juvenile biomass increases, while adult biomass decreases. In contrast and equally in line with our conclusion at the end of the previous section, for $q>1$, that is, when adults have a higher mass-specific biomass production rate than juveniles, juveniles dominate the population at low mortality. Under the latter conditions, increases in mortality translate into an increase in adult equilibrium biomass, whereas juvenile biomass decreases. The ontogenetic asymmetry in biomass production, which occurs for $q \neq 1$, thus translates into a positive relationship between stage-specific equilibrium biomass and overall mortality, either juvenile or adult biomass depending on whether $q<1$ or $q>1$. Below we shall say that biomass overcompensation occurs when there is at least one value of $m>0$ for which the equilibrium biomass is larger than the equilibrium biomass for $m=0$.

Figure 2 illustrates the dynamic consequences of ontogenetic asymmetry in mass-specific biomass production. In the case of ontogenetic symmetry in ingestion, biomass production and mortality, the model simplifies to the 2dimensional model in terms of total consumer biomass $B$ and resource biomass $R$ given by (5). The equilibrium in the latter model is stable for our choice 445 of the resource dynamics, $G(R)=\rho\left(R_{\max }-R\right)$. Ontogenetic asymmetry in biomass production, however, can give rise to stable population cycles of consumers and resources for both $q<1$ and $q>1$. Depending on the value of $q$ the dynamics of the population size structure is distinctly different. For $q<1$ juveniles have a higher mass-specific ingestion rate than adults and hence are 450 the main consumer stage driving the population cycle. This leads to a distinct cohort structure in the population as a pulse of newborn juveniles suppresses food availability for adult consumers and effectively precludes any consumer reproduction before the main part of the dominating juvenile cohort has matured. This gives rise to a dynamics in the consumer size structure which is dominated by a single extended cohort of individuals progressing through the juvenile size range. In contrast, for $q>1$ adults have a higher mass-specific ingestion rate than juveniles. As a consequence, reproduction takes place continuously throughout the cycle, but a cohort of individuals that matures to the adult stage suppresses food availability for juveniles and hence slows down juvenile growth in body size. Because of this temporary slowing down of ju- 

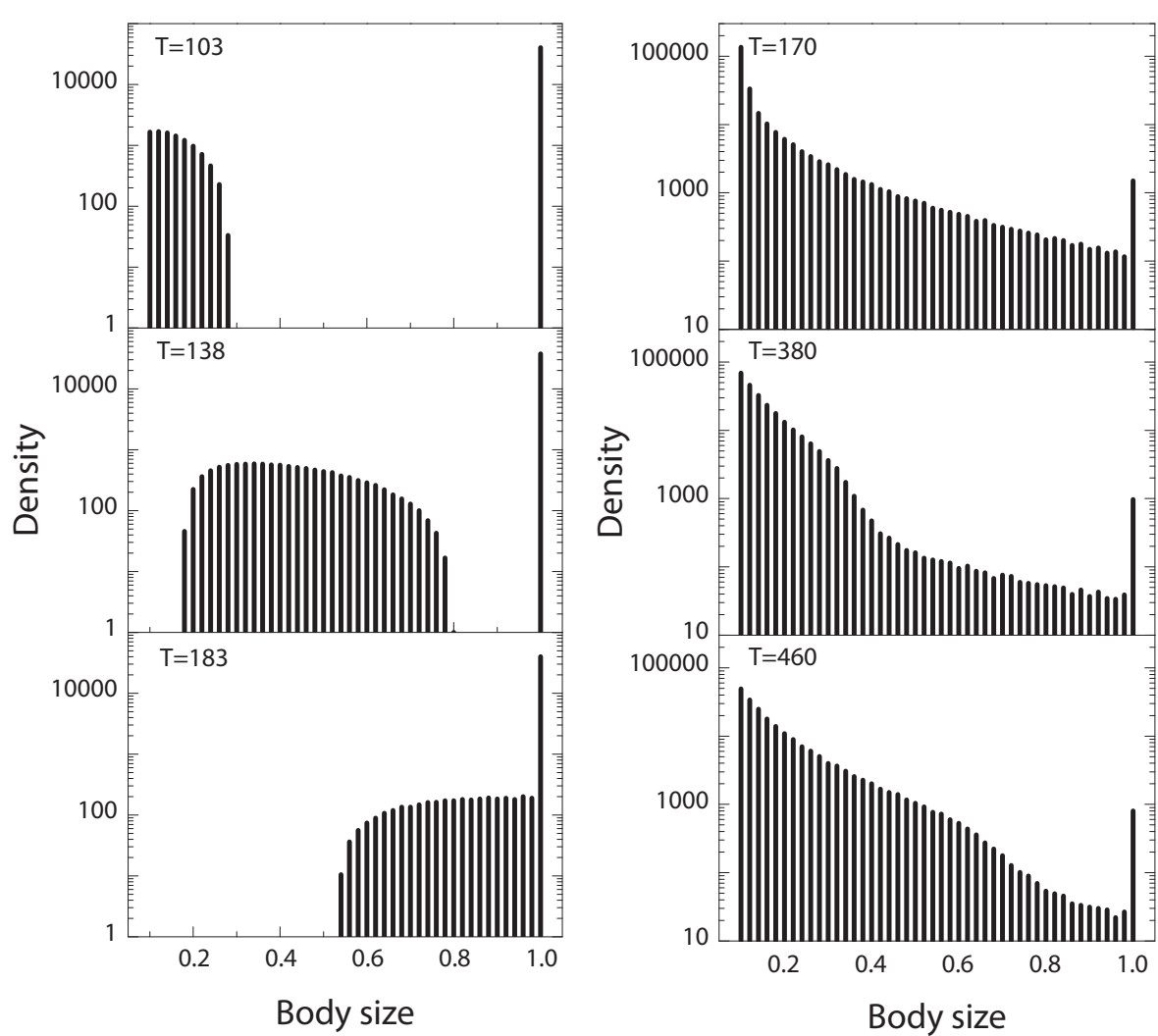

Fig. 2 Dynamics of the consumer size distribution in the stable limit cycle of consumers and resources for $q=0.4$ and $p=1.0$ (left, so-called juvenile-driven cohort-cycles) and $q=1.6$ and $p=1.0$ (right, adult-driven cohort-cycles).

venile growth a front builds up in the consumer distribution over the juvenile size range, which front slowly progresses to the maturation threshold.

\section{At which amount of asymmetry do the novel phenomena occur?}

Ontogenetic asymmetry in biomass production thus leads to two distinct sets of phenomena: overcompensation in juvenile biomass and juvenile-driven cohort cycles for $q<1$, and overcompensation in adult biomass and adult-driven cohort cycles for $q>1$ (assuming ontogenetic symmetry in mortality, $p=1$ ). To assess the dependence of these phenomena on the extent of asymmetry we analyzed (i) for which parameter combinations $p$ and $q$ equations (44), (46) and (47) predict an increase of juvenile or adult biomass with increasing mortality and (ii) for which parameter combinations $p$ and $q$ the equilibrium of the model (41) destabilizes through a Hopf bifurcation. The results of these calculations are shown in Figure 3 . 
To study the response of the equilibrium juvenile or adult biomass to ining, additional mortality (i.e. tuted $d_{J}+m$ and $d_{A}+m$ for $d_{J}$ and $d_{A}$, respectively, in Equations (44), 46 and (47) to get the (implicitly defined) functions $\tilde{R}: \mathbb{R}_{+} \rightarrow \mathbb{R}_{+}: m \mapsto \tilde{R}(m)$, $\tilde{J}: \mathbb{R}_{+} \rightarrow \mathbb{R}_{+}: m \mapsto \tilde{J}(m)$ and $\tilde{A}: \mathbb{R}_{+} \rightarrow \mathbb{R}_{+}: m \mapsto \tilde{A}(m)$, which we differentiated with respect to $m$ to get

$$
\begin{aligned}
& \frac{d \tilde{J}}{d m}(m)=\gamma_{0}+\gamma_{1} \frac{d \tilde{R}}{d m}(m) \\
& \frac{d \tilde{A}}{d m}(m)=\alpha_{0}+\alpha_{1} \frac{d \tilde{R}}{d m}(m)
\end{aligned}
$$

with $\gamma_{0}, \gamma_{1}, \alpha_{0}$ and $\alpha_{1}$ and the derivative $\frac{d \tilde{R}}{d m}(m)$, determined by the implicit differentiation of Equation (44), given in Appendix A.

As it turns out, juvenile biomass overcompensation always already occurs at the low end of the additional mortality $m$. Hence, for given values of the mortality asymmetry $p$ and otherwise default parameter values, we determined the limits to the occurrence of juvenile biomass overcompensation by solving Equation 44 for the equilibrium resource density $\tilde{R}$ together with $\frac{d \tilde{J}}{d m}(0)=0$ (using (48a)) for the equilibrium resource density $\tilde{R}$ and the threshold production asymmetry parameter $q$ using the Newton-Chord method with Broyden update (see Kuznetsov 1995, p. 418) and a standard continuation technique.

In the case of adult biomass overcompensation it may be that the overcompensation only starts at somewhat higher values of $m$. To delimit the set of parameter values for which at least some $m>0$ leads to equilibrium adult biomass densities higher than the density at $m=0$, we solved given a particular value of $p$ Equation (44) together with the same equation once again, but now with $d_{J}+\bar{m}$ and $\bar{d}_{A}+\bar{m}$ substituted for $d_{J}$ and $d_{A}$, respectively, the equation $\tilde{A}(0)=\tilde{A}(\bar{m})$ (using $(47)$ and the same equation once more with $d_{J}+\bar{m}$ and $d_{A}+\bar{m}$ substituted for $d_{J}$ and $d_{A}$, respectively) and $\frac{d \tilde{A}}{d m}(\bar{m})=0$ 500 (using $48 \mathrm{~b})$ ). These 4 equations determine the unknowns $\tilde{R}(0), \tilde{R}(\bar{m}), \bar{m}$ and $q$. The curve in the $(p, q)$-plane determined by this system of equations was computed using the same continuation technique as before.

Figure 3 shows the parameter regions for which juvenile or adult biomass overcompensation occurs, dependent on the mortality asymmetry $p$ and the biomass production asymmetry $q$. The occurrence of biomass overcompensation is mostly determined by the production asymmetry $q$ with significant influences of mortality asymmetry $p$ only for small $p$ values $(p \lesssim 0.4)$. For the case of ontogenetic symmetry in mortality $(p=1.0)$ these boundaries were also calculated as a function of the ratio between body size at birth and maturation, $z$, and the biomass production asymmetry $q$ (figure 3 right panel) showing that again the biomass production asymmetry mostly determines the occurrence of biomass overcompensation with only very small effects of the birth/maturation size ratio $z$.

Hopf bifurcation curves for model (41) were computed as a function of two 

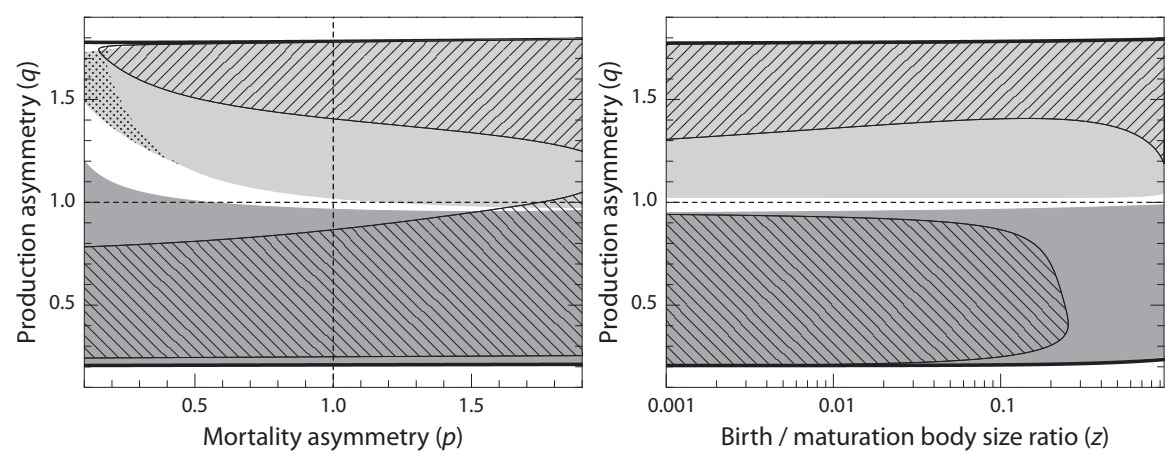

Fig. 3 Parameter combinations, for which overcompensation in equilibrium juvenile (dark grey regions) or adult biomass (light grey regions) occurs and for which juvenile-driven (cross-diagonally hatched regions) and adult-driven cohort-cycles (diagonally hatched regions) represent the stable attractor of model dynamics. In the dotted, light-grey parameter region at low $p$ and $q>1$ equilibrium adult biomass first decreases with increasing mortality before it increases above the equilibrium density at background mortality levels. In the right panel $p=1.0$.

Consider small perturbations to the equilibrium values $\tilde{R}, \tilde{J}$ and $\tilde{A}$ and to the equilibrium maturation rate $\tilde{\Psi}$, defined as:

$$
\tilde{\Psi}=\frac{\nu_{A}(\tilde{R}) \tilde{A} e^{-d_{J} \tilde{\tau}}}{z}
$$

and juvenile delay $\tilde{\tau}$ (equation 42 ):

$$
\begin{aligned}
& R(t)=\tilde{R}+\Delta_{R} e^{\lambda t} \\
& J(t)=\tilde{J}+\Delta_{J} e^{\lambda t} \\
& A(t)=\tilde{A}+\Delta_{A} e^{\lambda t} \\
& \Psi(t)=\tilde{\Psi}+\Delta_{\Psi} e^{\lambda t} \\
& \tau(t)=\tilde{\tau}+\Delta_{\tau} e^{\lambda t}
\end{aligned}
$$

Substitution of these relationships in equation (41e) leads after linearization to the following relationship between $\Delta_{\tau}$ and $\Delta_{R}$ :

$$
\Delta_{\tau}=\frac{\nu_{J}^{\prime}(\tilde{R})\left(e^{-\lambda \tilde{\tau}}-1\right)}{\nu_{J}(\tilde{R}) \lambda} \Delta_{R}
$$

Similarly, substitution of these relationships into equation (41d) leads after linearization to:

$$
\Delta_{\Psi}=\epsilon_{0} \tilde{A} \Delta_{R}+\epsilon_{1} \Delta_{A}
$$

${ }_{525}$ in which

$$
\begin{aligned}
& \epsilon_{0}=d_{A}\left(\frac{\nu_{J}^{\prime}(\tilde{R})}{\nu_{J}(\tilde{R})}\left(1+\frac{d_{J}}{\lambda}\right)\left(1-e^{-\lambda \tilde{\tau}}\right)+\frac{\nu_{A}^{\prime}(\tilde{R})}{\nu_{A}(\tilde{R})} e^{-\lambda \tilde{\tau}}\right) \\
& \epsilon_{1}=d_{A} e^{-\lambda \tilde{\tau}}
\end{aligned}
$$


These expressions for $\epsilon_{0}$ and $\epsilon_{1}$ have been simplified using the identities $\tilde{\Psi}=$ $d_{A} \tilde{A}$ (from Equation (41b) ) and $\nu_{A}(\tilde{R}) e^{-d_{J} \tilde{\tau}}=z d_{A}$ (from Equation (44)).

Substitution of the perturbations (50)-(54) into equations (41a)- (41c) subsequently lead together with the expressions (55) and (56) for $\Delta_{\tau}$ and $\Delta_{\Psi}$, respectively, to the following matrix equation:

$$
\mathbf{K}(\tilde{R}, \lambda)\left(\begin{array}{c}
\Delta_{R} \\
\Delta_{J} \\
\Delta_{A}
\end{array}\right)=0
$$

with $\mathbf{K}(\lambda)$ defined as:

$$
535 \quad \mathbf{K}(\tilde{R}, \lambda)=\left(\begin{array}{ccc}
G^{\prime}(\tilde{R})-I_{J}^{\prime}(\tilde{R}) \tilde{J}-I_{A}^{\prime}(\tilde{R}) \tilde{A}-\lambda & -I_{J}(\tilde{R}) & -I_{A}(\tilde{R}) \\
\nu_{J}^{\prime}(\tilde{R}) \tilde{J}+\nu_{A}^{\prime}(\tilde{R}) \tilde{A}-\epsilon_{0} \tilde{A} & \nu_{J}(\tilde{R})-d_{J}-\lambda & \nu_{A}(\tilde{R})-\epsilon_{1} \\
\epsilon_{0} \tilde{A} & 0 & \epsilon_{1}-d_{A}-\lambda
\end{array}\right)
$$

Note that $\tilde{J}, \tilde{A}$ and $\tilde{\tau}$ in this matrix are explicit expressions in terms of the equilibrium resource density $\tilde{R}$, given by equation $(46, \sqrt{47}$ and $\sqrt{42}$, respectively.

Hopf bifurcation boundaries are now determined by the equilibrium condition (44) for the equilibrium resource density together with the complex-valued equation:

$$
\operatorname{det} \mathbf{K}(\tilde{R}, \imath \theta)=0
$$

which determines a pair of purely imaginary roots $\lambda= \pm \imath \theta$ of the characteristic equation (57). Solutions as a function of two parameters were obtained by Newton iteration and continuation, as described before for the computation of the limits to juvenile and adult biomass overcompensation.

Figure 3 shows that by and large the equilibrium of model (41) is unstable and stable limit cycles occur both for $q<1$ and for $q>1$, but not for ontogenetic symmetry in biomass production $(q=1)$ except when mortality is strongly skewed toward adult consumers $(p \gtrsim 1.6)$. The two distinct regions of parameters, for which limit cycles occur, were identified on the basis of numerical integrations of the model as representing juvenile-driven $(q<1)$ and adult-driven cohort cycles $(q>1$, see Figure 2). Except when mortality is strongly skewed toward adult consumers $(p \gtrsim 1.6)$ the two regions of parameters with stable limit cycles are contained within the parameter regions, for which juvenile and adult biomass overcompensation occurs, but are substantially smaller. Ontogenetic asymmetry in biomass production is hence less likely to induce dynamic effects (cohort cycles) than equilibrium effects (biomass overcompensation). The right panel of Figure 3 furthermore shows that the juvenile-driven cycles no longer occur for larger ratios of the body size at birth and at maturation $(z \gtrsim 0.25)$. Otherwise the effect of $z$ on the occurrence of these cycles is small, analogous to its effect on the occurrence of biomass overcompensation. 


\section{Concluding remarks}

paper we analyzed the relationship between size-structured population models and their unstructured counterparts that model dynamics solely on the basis of total population biomass. This led to the following take-home messages.

- In case of ontogenetic symmetry in mass-specific ingestion, mass-specific biomass production and mortality the dynamics of total population biomass decouples from the dynamics of the population structure. Assuming ontogenetic symmetry is the only biologically plausible way to assure this effect.

- In case of ontogenetic symmetry the equilibrium size distribution is always the same, independent of external conditions such as mortality or resource productivity.

- In case of ontogenetic symmetry the net production of new biomass through somatic growth or reproduction in every size range of consumers exactly equals the loss rate through mortality in that size range. In particular, in equilibrium both the juvenile and adult stage are zero net-producers of biomass.

- In contrast, with deviations from ontogenetic symmetry, if in equilibrium the juvenile stage becomes a net production stage of biomass juveniles make up a smaller proportion of total population biomass (juvenile biomass is underrepresented) than expected when ontogenetic symmetry applies. Vice versa, if in equilibrium the juvenile stage becomes a net loss stage of biomass juvenile biomass is overrepresented in the population, compared to when ontogenetic symmetry applies.

- On embedding a model with ontogenetic symmetry in a larger model family also allowing ontogenetic asymmetry, the previous two conclusions can be violated even for small deviations from the ontogenetically symmetric case (in the particular model that we studied $5 \%$ or even less difference between juveniles and adults in mass-specific biomass productions was enough).

- In case of ontogenetic asymmetry two different domains could be distinguished, in which the conclusions for the ontogenetically symmetric case no longer hold good, with asymmetry in mass-specific biomass production being the main variable along which the domains were separated:

- When juveniles have a higher mass-specific biomass production than adults, juvenile biomass overcompensation occurs next to juvenile-driven cycles.

600

- Vice versa, when adults have a higher mass-specific biomass production relative to juveniles, adult biomass overcompensation occurs next to adult-driven cycles.

We have considered forms of ontogenetic asymmetry in mass-specific net production of biomass that arise from factors intrinsic to the individual consumer, in particular from differences in mass-specific maximum ingestion rate. Ontogenetic asymmetry in biomass production can, however, also arise from extrinsic factors, for example, when juveniles and adults feed on different re- 
sources and the availabilities of these resources differ. In case of such extrinsically induced ontogenetic asymmetry the biomass production of juveniles and adults are decoupled and competition for resources between the stages is absent. Like asymmetry induced by factors intrinsic to the individual, such asymmetry induced by different resource availabilities have been shown also to result in juvenile biomass overcompensation and juvenile-driven cycles, when resource availability is higher for juveniles than for adults, whereas adult biomass overcompensation and adult-driven cycles may result when resource availability is higher for adults than for juveniles (De Roos and Persson 2013).

In view of the dedication of this paper we highlight the contribution of Odo Diekmann to the insights presented in this paper, which pertain to the occurrence of different types of population cycles in case of ontogenetic asymmetry. In Diekmann et al (2010) it was shown using a general size-structured model that the maturation delay on its own does not lead to oscillations. More specifically, if adults and juveniles only differ in that juveniles convert substrate into growth and adults convert it into offspring, with fixed conversion factors, then the stability properties of the size-structured model exactly mimic those of an unstructured model. Population cycles hence do not occur with semi-chemostat resource dynamics if there are no differences between juvenile and adult consumers in either resource ingestion rate or mortality. This result correspond to our result that the size-structured model simplifies to an unstructured model in case of ontogenetic symmetry in net biomass produc${ }_{630}$ tion and mortality. Diekmann et al (2010) furthermore analyzed the special case when only juveniles competed for the resource while adult reproduction was constant, in which case limit cycles occurred with a period between one and two times the juvenile period. This result agrees with our result that adult-driven cohort cycles occur in case of ontogenetic asymmetry with adults having a larger net biomass production rate than juveniles. Diekmann et al (2010) also found that population cycles could occur when only adults compete for the resource and juveniles hence have a larger net biomass production rate, which is in line with our prediction that juvenile-driven cohort cycles occur under such conditions. However, the period of the cycles identified by Diekmann 640 et al (2010) was between two and four times the juvenile period, whereas the cycle period of juvenile-driven cohort cycles is between one and two times the juvenile period. The cycles found by Diekmann et al (2010) hence represent socalled "delayed-feedback cycles" as opposed to the "single-generation" cycles that we identified (Gurney and Nisbet 1985). This discrepancy may. however, have resulted from the assumption in Diekmann et al (2010) of quasi-steadystate dynamics of the resource density, which has been shown to potentially lead to the disappearance of population cycles (De Roos and Persson 2003).

Acknowledgements L. Persson is financially supported by grants from the Swedish Research Council and the Swedish Research Council for Environment, Agricultural Sciences 650 and Spatial Planning. The work of Hans Metz benefitted from the support from the "Chair Modélisation Mathématique et Biodiversité of Veolia Environnement-Ecole PolytechniqueMuseum National d'Histoire Naturelle-Fondation X". 


\section{A The explicit expressions for $\gamma_{i}$ and $\alpha_{i}$ and $\frac{d \tilde{R}}{d m}$}

In this appendix we give the missing ingredients of formulas 48a and 48b.

$$
\begin{aligned}
& \gamma_{0}=\frac{G(\tilde{R})\left(\nu_{A}(\tilde{R})-d_{A}-m\right)\left(I_{J}(\tilde{R})-I_{A}(\tilde{R})\right)}{\left(I_{J}(\tilde{R})\left(\nu_{A}(\tilde{R})-d_{A}-m\right)-I_{A}(\tilde{R})\left(\nu_{J}(\tilde{R})-d_{J}-m\right)\right)^{2}} \\
& -\frac{G(\tilde{R})}{I_{J}(\tilde{R})\left(\nu_{A}(\tilde{R})-d_{A}-m\right)-I_{A}(\tilde{R})\left(\nu_{J}(\tilde{R})-d_{J}-m\right)} \\
& \gamma_{1}=\frac{G^{\prime}(\tilde{R})\left(\nu_{A}(\tilde{R})-d_{A}-m\right)+G(\tilde{R}) \nu_{A}^{\prime}(\tilde{R})}{I_{J}(\tilde{R})\left(\nu_{A}(\tilde{R})-d_{A}-m\right)-I_{A}(\tilde{R})\left(\nu_{J}(\tilde{R})-d_{J}-m\right)} \\
& -\frac{G(\tilde{R})\left(\nu_{A}(\tilde{R})-d_{A}-m\right)^{2} I_{J}^{\prime}(\tilde{R})}{\left(I_{J}(\tilde{R})\left(\nu_{A}(\tilde{R})-d_{A}-m\right)-I_{A}(\tilde{R})\left(\nu_{J}(\tilde{R})-d_{J}-m\right)\right)^{2}} \\
& +\frac{G(\tilde{R})\left(\nu_{A}(\tilde{R})-d_{A}-m\right) I_{A}^{\prime}(\tilde{R})\left(\nu_{J}(\tilde{R})-d_{J}-m\right)}{\left(I_{J}(\tilde{R})\left(\nu_{A}(\tilde{R})-d_{A}-m\right)-I_{A}(\tilde{R})\left(\nu_{J}(\tilde{R})-d_{J}-m\right)\right)^{2}} \\
& -\frac{G(\tilde{R})\left(\nu_{A}(\tilde{R})-d_{A}-m\right)\left(I_{J}(\tilde{R}) \nu_{A}^{\prime}(\tilde{R})-I_{A}(\tilde{R}) \nu_{J}^{\prime}(\tilde{R})\right)}{\left(I_{J}(\tilde{R})\left(\nu_{A}(\tilde{R})-d_{A}-m\right)-I_{A}(\tilde{R})\left(\nu_{J}(\tilde{R})-d_{J}-m\right)\right)^{2}} \\
& \alpha_{0}=\frac{G(\tilde{R})\left(\nu_{J}(\tilde{R})-d_{J}-m\right)\left(I_{A}(\tilde{R})-I_{J}(\tilde{R})\right)}{\left(I_{A}(\tilde{R})\left(\nu_{J}(\tilde{R})-d_{J}-m\right)-I_{J}(\tilde{R})\left(\nu_{A}(\tilde{R})-d_{A}-m\right)\right)^{2}} \\
& -\frac{G(\tilde{R})}{I_{A}(\tilde{R})\left(\nu_{J}(\tilde{R})-d_{J}-m\right)-I_{J}(\tilde{R})\left(\nu_{A}(\tilde{R})-d_{A}-m\right)} \\
& \alpha_{1}=\frac{G^{\prime}(\tilde{R})\left(\nu_{J}(\tilde{R})-d_{J}-m\right)+G(\tilde{R}) \nu_{J}^{\prime}(\tilde{R})}{I_{A}(\tilde{R})\left(\nu_{J}(\tilde{R})-d_{J}-m\right)-I_{J}(\tilde{R})\left(\nu_{A}(\tilde{R})-d_{A}-m\right)} \\
& -\frac{G(\tilde{R})\left(\nu_{J}(\tilde{R})-d_{J}-m\right)^{2} I_{A}^{\prime}(\tilde{R})}{\left(I_{A}(\tilde{R})\left(\nu_{J}(\tilde{R})-d_{J}-m\right)-I_{J}(\tilde{R})\left(\nu_{A}(\tilde{R})-d_{A}-m\right)\right)^{2}} \\
& +\frac{G(\tilde{R})\left(\nu_{J}(\tilde{R})-d_{J}-m\right) I_{J}^{\prime}(\tilde{R})\left(\nu_{A}(\tilde{R})-d_{A}-m\right)}{\left(I_{A}(\tilde{R})\left(\nu_{J}(\tilde{R})-d_{J}-m\right)-I_{J}(\tilde{R})\left(\nu_{A}(\tilde{R})-d_{A}-m\right)\right)^{2}} \\
& -\frac{G(\tilde{R})\left(\nu_{J}(\tilde{R})-d_{J}-m\right)\left(I_{A}(\tilde{R}) \nu_{J}^{\prime}(\tilde{R})-I_{J}(\tilde{R}) \nu_{A}^{\prime}(\tilde{R})\right)}{\left(I_{A}(\tilde{R})\left(\nu_{J}(\tilde{R})-d_{J}-m\right)-I_{J}(\tilde{R})\left(\nu_{A}(\tilde{R})-d_{A}-m\right)\right)^{2}}
\end{aligned}
$$


Determinig the derivative $d \tilde{R}(m) / d m$ using the implicit function theorem after substitution of $d_{J}+m$ and $d_{A}+m$ for $d_{J}$ and $d_{A}$, respectively, in equation 44 leads to

$$
\begin{gathered}
\frac{\nu_{A}(\tilde{R}) z^{\left(d_{J}+m\right) / \nu_{J}(\tilde{R})-1}}{d_{A}+m}\left(\frac{\nu_{A}^{\prime}(\tilde{R})}{\nu_{A}(\tilde{R})}-\frac{\left(d_{J}+m\right) \ln (z) \nu_{J}^{\prime}(\tilde{R})}{\left(\nu_{J}(\tilde{R})\right)^{2}}\right) \frac{d \tilde{R}}{d m}(m) \\
+\frac{\nu_{A}(\tilde{R}) z^{\left(d_{J}+m\right) / \nu_{J}(\tilde{R})-1}}{d_{A}+m}\left(\frac{\ln (z)}{\nu_{J}(\tilde{R})}-\frac{1}{d_{A}+m}\right)=0 \Rightarrow \\
\frac{d \tilde{R}}{d m}(m)=\left(\frac{1}{d_{A}+m}-\frac{\ln (z)}{\nu_{J}(\tilde{R})}\right)\left(\frac{\nu_{A}^{\prime}(\tilde{R})}{\nu_{A}(\tilde{R})}-\frac{\left(d_{J}+m\right) \ln (z) \nu_{J}^{\prime}(\tilde{R})}{\left(\nu_{J}(\tilde{R})\right)^{2}}\right)^{-1}
\end{gathered}
$$

\section{References}

Andersen KH, Beyer JE (2006) Asymptotic size determines species abundance in the marine size spectrum. American Naturalist 168(1):54-61

Anderson EC, Bell GI, Petersen DF, Tobey RA (1969) Cell growth and division iv. determination of volume growth rate and division probability. Biophysical Journal 9:246-263

Begon M, Harper JL, Townsend CR (1996) Ecology: Individuals, populations and communities. 3rd edition. Blackwell Scientific Publications, Oxford

Bell GI (1968) Cell growth and division ii. conditions for balanced exponential growth in a mathematical model. Biophysical Journal 8:431-444

Bell GI, Anderson EC (1967) Cell growth and division. i. a mathematical model with applications to cell volume distributions in mammalian suspension cultures. Biophys J $7: 329-351$

Brose U, Williams RJ, Martinez ND (2006) Allometric scaling enhances stability in complex food webs. Ecology Letters 9(11):1228-1236

Caswell H (2001) Matrix population models - Construction, analysis and interpretation, 2nd edn. Sinauer Associates, Sunderland, MA

De Roos AM (1988) Numerical methods for structured population models: The escalator boxcar train. Numerical Methods for Partial Differential Equations 4:173-195

De Roos AM (1997) A gentle introduction to physiologically structured population models. In: Tuljapurkar S, Caswell H (eds) Structured population models in marine, terrestrial and freshwater systems, Chapman-Hall, New York, pp 119-204

De Roos AM, Persson L (2003) Competition in size-structured populations: Mechanisms inducing cohort formation and population cycles. Theoretical Population Biology 63(1):116

De Roos AM, Persson L (2013) Population and community ecology of ontogenetic development. Monographs in Population Biology 51, Princeton University Press, Princeton

De Roos AM, Metz JAJ, Evers E, Leipoldt A (1990) A size dependent predator-prey interaction: Who pursues whom? Journal of Mathematical Biology 28:609-643

De Roos AM, Schellekens T, Van Kooten T, Van De Wolfshaar K, Claessen D, Persson L (2007) Food-dependent growth leads to overcompensation in stage-specific biomass when mortality increases: The influence of maturation versus reproduction regulation. American Naturalist 170:E59-E76

De Roos AM, Schellekens T, Van Kooten T, Van De Wolfshaar K, Claessen D, Persson L (2008) Simplifying a physiologically structured population model to a stage-structured biomass model. Theoretical Population Biology 73(1):47-62

Diekmann O, Gyllenberg M, Metz JAJ, Thieme HR (1998) On the formulation and analysis of general deterministic structured population models - i. linear theory. Journal of Mathematical Biology 36(4):349-388 
Diekmann O, Gyllenberg M, Metz JAJ (2003) Steady-state analysis of structured population models. Theoretical Population Biology 63(4):309-338

Diekmann O, Gyllenberg M, Metz JAJ, Nakaoka S, de Roos AM (2010) Daphnia revisited: Local stability and bifurcation theory for physiologically structured population models explained by way of an example. Journal of Mathematical Biology 61(2):277-318

Fredrickson AG, Ramkrishna D, Tsuchiya HM (1967) Statistics and dynamics of procaryotic cell populations. Mathematical Biosciences 1:327-374

Gurney WSC, Nisbet RM (1985) Fluctuation periodicity, generation separation, and the expression of larval competition. Theoretical Population Biology 28(2):150-180

Kirkilionis MA, Diekmann O, Lisser B, Nool M, Sommeijer B, De Roos AM (2001) Numerical continuation of equilibria of physiologically structured population models. i. theory. Mathematical Models \& Methods In Applied Sciences 11(6):1101-1127

Kuznetsov YA (1995) Elements of applied bifurcation theory. Springer-Verlag, Heidelberg

McCann K, Hastings A, Huxel GR (1998) Weak trophic interactions and the balance of nature. Nature 395(6704):794-798

McKendrick AG (1926) Application of mathematics to medical problems. Proceedings of the Edinburgh Mathematical Society 44:98-130

Metz JAJ, Diekmann O (1986) The dynamics of physiologically structured populations, Lecture Notes in Biomathematics, vol 68. Springer-Verlag, Heidelberg

Metz JAJ, Diekmann O (1991) Models for physiologically structured populations. i. the abstract foundation of linear chain trickery. Lecture Notes in Pure and Appl Math 133:269-289

Murphy LF (1983) A nonlinear growth mechanism in size structured population dynamics. Journal of Theoretical Biology 104:493-506

Nisbet RM, Gurney WSC (1983) The systematic formulation of population models for insects with dynamically varying instar duration. Theoretical Population Biology 23(1):114-135

Sheldon R, Prakash A, Sutcliffe W (1972) The size distribution of particles in the ocean. Limnology and Oceanography 17:327-340

Sinko JW, Streifer W (1967) A new model for age-size structure of a population. Ecology 48:910-918

Sinko JW, Streifer W (1969) Applying models incorporating age-size structure of a population to daphnia. Ecology 50:608-615

Sinko JW, Streifer W (1971) A model for populations reproducing by fission. Ecology 52:330335

Tsuchiya HM, Fredrickson AG, Aris P (1966) Dynamics of microbial cell populations. Advances in Chemical Engineering 6:125-198

VanSickle J (1977) Analysis of a distributed-parameter population model based on physiological age. Journal Theoretical BioIogy 64:571-586

VonFoerster H (1959) Some remarks on changing populations. In: Stohlman F (ed) The Kinetics of Cellular Proliferation, Grune and Stratton, New York

Yodzis P, Innes S (1992) Body size and consumer resource dynamics. American Naturalist 139:1151-1175 